\title{
FACTORES QUE INCIDEN EN LA EROSIÓN HÍDRICA
}

\section{FACTORS INFLUENCING WATER EROSION}

Virginia Alvarado García ${ }^{1}$

htpps://doi.org/10.52109/cyp2021217

1 Laboratorio de Vida Silvestre y Salud, Universidad Estatal a Distancia, Costa Rica, valvaradog@uned.ac.cr, https://orcid.org/0000-0001-7430-0549.

\section{REGISTROS}

Recibido el 14/05/2021

Aceptado el 25/07/2021

Publicado el 31/07/2021

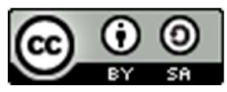

\section{PALABRAS CLAVE}

Erosividad, erodabilidad, degradación, suelo, conservación.

\section{KEYWORDS}

Erosivity, erodibility, degradation, soil, conservation.

\section{RESUMEN}

La erosión es el resultado de la interacción de diversos factores que varían espaciotemporalmente. Su estudio debe estar enfocado en la comprensión de los elementos que inciden en dicho proceso, para así, lograr un abordaje adecuado en el manejo y conservación del suelo. Esta revisión pretende analizar los principales factores que condicionan la erosión hídrica del suelo y la influencia de cada uno de ellos; además de presentar algunas técnicas de conservación de suelos. Entre los más importantes se pueden citar, la precipitación, la cobertura vegetal, la topografía, las características edáficas y los factores antrópicos. La pérdida de este recurso se da más comúnmente en sitios sin cobertura vegetal, con un uso de suelo inadecuado, zonas expuestas a intensas lluvias, pendientes fuertes y de largas longitudes, y falta o ausencia de prácticas preventivas. El uso de tecnologías como los abonos orgánicos, los cultivos de cobertura, las barreras vivas, las cortinas rompevientos, la bioingeniería y la revegetación, son clave para una controlar el proceso erosivo y aumentar la productividad de las tierras. : Por ello, la estimación de la resistencia de los suelos a la erosión debe ser integral; dado que le proceso erosivo no depende en una sola variable, sino de la combinación de componentes que, a su vez, inciden en la magnitud de sus efectos.

\section{ABSTRACT}

Erosion is the result of the interaction of several factors that vary in space and time. Its study should be focused on understanding the elements that affect this process, in order to achieve an adequate approach in soil management and conservation. This overview aims to analyze the main factors that condition soil water erosion and the influence of each of them; in addition to presenting some soil conservation techniques. The most important are, precipitation, vegetation cover, topography, edaphic characteristics and anthropic factors. The loss of this resource occurs more commonly in sites without vegetation cover, inadequate land use, areas exposed to intense rains, steep slopes and long lengths, and lack or absence of preventive practices. The use of technologies such as organic fertilizers, cover crops, live barriers, windbreaks, bioengineering and revegetation, are key to control erosion process and increase land productivity. For this reason, the estimation of soil resistance to erosion must be comprehensive; since the erosive process does not depend on one variable, but on the combination of components that, in turn, affect the magnitude of its effects. 


\section{INTRODUCCIÓN}

La erosión del suelo es un proceso complejo y dinámico; el cual representa uno de los mayores problemas de degradación de tierras a nivel mundial. Afecta no solo las características geomorfológicas de un terreno; sino que, además, modifica su fertilidad, su productividad, y la calidad y cantidad de otros recursos naturales. A su vez, la tasa de erosión depende de una serie de factores geomorfológicos, edáficos, régimen hidrológico, cobertura vegetal, uso del suelo, entre otros (Aiello, Adamo y Canora, 2015; Blanco Chávez, 2019).

Este proceso de desgaste conlleva la pérdida, desprendimiento y transporte del material del suelo por acción del agua o del viento; siendo la precipitación, el factor más influyente debido al mecanismo de salpicadura, que es el principal agente iniciador de la erosión (Alvarado-García y Zúñiga-Amador, 2020).

El potencial erosivo de la lluvia determina en gran medida, su erodabilidad. Esta susceptibilidad al desprendimiento y transporte de las partículas del suelo está influenciada por numerosas propiedades extrínsecas e intrínsecas (Pérez-Salinas, Rodríguez, López-Santos, Torres-González, Meraz-Jiménez, Díaz-Romo y Valdivia-Martínez, 2019), y representan el grado de resistencia natural que ofrece el suelo a ser erosionado (Ramírez-Ortiz, Hincapié-Gómez y Sadeghian-Khalajabadi, 2009).

Esta sensibilidad o resistencia a la erosión evidencia que diferentes tipos de suelo se erosionan a diferentes tasas, cuando los factores que la afectan son los mismos; es decir, los factores erosivos determinan la magnitud de los agentes erosivos (Ramírez-Ortiz et al., 2009). Por ello, y dado que, la causa de la erosión no radica en un solo componente, esta revisión pretende analizar los principales elementos que intervienen en dicho proceso.

Factores

\section{Precipitación}

En función de la causa, la erosión hídrica es la más común y es acelerada por la acción de las gotas de lluvia sobre un terreno desnudo. Cuando la gota cae por gravedad, impacta la superficie de forma directa con una fuerza considerable, lo que disgrega las partículas del suelo, las arrastra y finalmente las deposita (Suárez, 2001; Imeson y Curfs, 2008; Ragonessi y Soto, 2010).

Según Suárez (1980), la precipitación es el factor más influyente en tales procesos, a causa principalmente del mecanismo de salpicadura, el cual constituye el principal agente iniciador de la erosión. El poder erosivo de las gotas de lluvia depende no solamente de las propiedades del suelo, sino también de la carga energética (tamaño y velocidad) con la que impacta la superficie y de la presencia de 
vegetación. Cuando existe un sistema foliar que intercepta las gotas, se reduce el tamaño y velocidad de la gota (León, 2001; Suárez, 2001).

La erosividad o agresividad de la lluvia sobre el suelo, representa la energía cinética con que las gotas de lluvia a determinada intensidad pueden romper los agregados superficiales en partículas de tamaño transportable. Es, por lo tanto, un atributo complejo, ya que relaciona cantidad y duración de las lluvias, y diámetro y velocidad de las gotas de lluvia (Colotti Bizzarri, 1999).

La intensidad representa el volumen de lluvia caída por unidad de tiempo. Se estima que todo evento pluvial con intensidad menor a $25 \mathrm{~mm} \mathrm{~h}-1$ no se considera erosivo (Núñez, 2010); sin embargo, esto va a depender de la duración de las lluvias, ya que esto, va a regular la cantidad de agua caída sobre el suelo. Las precipitaciones largas son más erosivas que las de corta duración, ya que el terreno supera su capacidad de infiltración y produce lluvia en exceso o escorrentía (Alvarado-García, 2020).

Adicionalmente, la distribución de intensidades dentro de una tormenta determina en gran medida, el comportamiento de la escorrentía. Si las intensidades máximas ocurren al inicio de un aguacero, gran parte será infiltrada y otra encauzada hacia alguna red de drenaje; mientras que, si esto ocurre al final del aguacero, cuando el suelo está saturado y la infiltración es mínima, el volumen de agua será mayor (Robredo-Sánchez 2014).

En términos de erosión, el porcentaje del agua de lluvia que se convierte en agua de escorrentía es fundamental para la toma de decisiones. En función de ciertos parámetros edáficos, se obtiene un coeficiente de escorrentía; el cual aumentará, al incrementar la impermeabilidad del suelo y la pendiente, y disminuir la cobertura vegetal (Alvarado-García, 2020).

En eventos de alta intensidad de lluvia, ésta posee gran capacidad de transporte y arrastre de elementos finos y gruesos (Almoza, Medina, Schiettecatte, Ruiz y Leal, 2007); lo cual, provoca un reordenamiento de la estructura edáfica a lo largo de un terreno y la formación de capas gruesas, menos porosas e impermeables, que aumentan la escorrentía superficial y la exposición del suelo (Robinson y Woodun, 2008).

\section{Vegetación}

El papel de la cobertura vegetal es imprescindible para la protección del suelo a nivel subterráneo y superficial. Amortigua el impacto de las gotas de lluvia, facilita la infiltración y frena la escorrentía (Alvarado-García y Zúñiga-Amador, 2020).

La vegetación es fundamental para la salud del suelo, sobre todo por el aporte de materia orgánica, la cual mantiene las características del suelo homogéneas (García, 2004), permite una mayor y mejor agregación de las partículas del suelo y provee mayor estabilidad estructural y resistencia a la erosión (Ramírez-Ortiz et al., 2009). 
El sistema radicular tiene implicaciones sobre la erosión; ya que, generalmente, las especies herbáceas presentan una alta densidad de raíces finas en los horizontes superficiales; en tanto que, las especies leñosas, se distribuyen más ampliamente en el perfil del suelo. Ambas le confieren al terreno, resistencia estructural y representan una excelente protección contra los impactos de la erosión (SepúlvedaLozada, Geissen, Ochoa-Gaona, Jarquín-Sánchez, Hernández de la Cruz, Capetillo y Zamora-Cornelio, 2009).

Estudios realizados por Dlamini, Orchard, Jewitt, Lorentz, Titshall y Chaplot (2011), Durán, Rodríguez, Martin, de Graaff, Francia y Flanagan (2011), Alvarado, Bermúdez, Romero y Piedra (2013) y Alvarado-García y Zúñiga-Amador (2020), concuerdan en que el uso de coberturas ayuda a disminuir la pérdida de suelo.

Topografía

Los principales factores topográficos que tienen influencia sobre la erosión hídrica son el ángulo de la pendiente, el largo y forma de la ladera (Gayoso y Alarcón, 1999). A mayor inclinación y longitud de la ladera, mayor severidad de la erosión (Dumas Salazar, 2012).

La longitud e inclinación de la pendiente están directamente relacionadas con la vulnerabilidad de los suelos a la erosión, a medida que se incrementa la inclinación y la longitud de la pendiente (Hincapié Gómez y Ramírez Ortíz, 2010), se incrementa el arrastre y depósito de sedimentos aguas abajo por acción de la escorrentía superficial (Suárez, 2001; Hernández, 2011).

En una ladera, la erosión del suelo no se da igual en toda su longitud. La erosión incrementa a medida que aumenta la pendiente del terreno, ya que también lo hacen la cantidad y la energía de la escorrentía superficial. En una superficie plana, las gotas de lluvia salpican las partículas de suelo en todas direcciones (Hernández, 2011); mientras que, en un terreno inclinado la erosión hídrica causa un máximo de pérdida de suelo en las áreas medias y bajas de la pendiente, y una acumulación de material en la base de las laderas (Mehuys, Tiessen, Villatoro, Sancho y Lobb, 2009).

Suelo

La erodabilidad representa el grado de sensibilidad frente a la erosión, y depende de sus características físicas y del manejo que se le dé a éste (Núñez, 2010). Según Henríquez y Cabalceta (1999) algunas propiedades físicas del suelo que inciden en el proceso erosivo son, la densidad aparente, densidad de partículas, espacio aéreo, porosidad total, infiltración y conductividad hidráulica; todas relacionadas con la textura del suelo y agentes formadores.

El suelo posee algunos atributos que aceleran el proceso erosivo, estas son: 
- Compactación: es un empaquetamiento de las partículas que constituyen la fracción sólida del suelo, lo que provoca una disminución del espacio poroso total y un aumento de la densidad aparente. Además, esto restringe el desarrollo radical de la vegetación (Henríquez, Ortiz, Largaespada, Portuguéz, Vargas, Villalobos y Gómez, 2011).

- Textura: los suelos con alto porcentaje de limo son más vulnerables a la erosión. Altos contenidos de arcilla y poca porosidad puede ocasionar un decremento en la velocidad de infiltración de agua en el suelo; en tanto que, los suelos arenosos son más resistentes al impacto de las gotas de lluvia, siempre que el suelo no esté saturado (Núñez, 2010).

- Estructura: un adecuado desarrollo estructural en los perfiles edáficos favorece la infiltración y disminuye la esocrrentía. Esto se da en suelos franco arcillosos, franco arcillo limosos, arcillosos y arcillo limosos (Núñez, 2010). El contenido de materia orgánica influye en la agregación de partículas de suelo y estabilidad de los agregados, lo cual favorece la resistencia del suelo a la erosión (Ramírez-Ortiz et al., 2009)

- Densidad aparente y porosidad: una alta densidad aparente posee menor porosidad. El predominio de mesoporos y macroporos promueve la infiltración y evita una acelerada saturación (Núñez, 2010). Esto concuerda con Ramírez-Ortiz et al. (2009), donde la densidad aparente, la estabilidad estructural y la conductividad hidráulica mostraron relación con la resistencia del suelo; por lo tanto, una menor densidad aparente le confiere al suelo mayor resistencia a la erosión y mayor estabilidad estructural.

- Conductividad hidráulica: la erodabilidad y la conductividad hidráulica son inversamente proporcionales; esta relación indica que la estructura subsuperficial determina el movimiento de agua en el perfil del suelo (Ramírez-Ortiz et al., 2009) y, por lo tanto, la permeabilidad de los horizontes.

- Pedregosidad: esta propiedad puede ser ambivalente, ya que las piedras frenan la infiltración directa de las gotas de lluvia; sin embargo, también pueden aumentarla, si toman la energía cinética de la lluvia. Las zonas pedregosas, en general, poseen una macroporosidad importante, que permite la infiltración y la conductividad hidráulica (Viramontes, Descroix y Bollery, 2006).

Uso

Las actividades humanas desencadenan una serie de procesos que reducen la capacidad actual o futura del suelo para funcionar y sostener los ecosistemas; para mantener o mejorar la calidad de los recursos naturales; y para para preservar la salud humana. Por lo tanto, la degradación del suelo es toda modificación que incida en la pérdida de las funciones de éste (Muñoz Iniesta, Ferreira Ramírez, Escalante Arriaga y López García, 2013). 
Los cambios significativos de la cubierta terrestre afectan sustancialmente la calidad y salud de los suelos. Un ejemplo de ello, son las actividades agrícolas en expansión y sus procesos de producción altamente mecanizados, los cuales provocan una mayor vulnerabilidad a inundaciones y problemas ambientales asociados, debido al aporte de sedimentos en los cuerpos de agua y a la transformación del relieve (Álvarez Rojas y Peña, 2013).

Las actividades humanas alteran fácilmente las características edáficas, lo que modifica a su vez, la respuesta hidráulica (Viramontes et al., 2006) e hidrológica de una cuenca (Alvarado García, 2020). Existe una relación proporcional entre la cobertura urbana y el uso de suelo; para un mismo evento de lluvia, el volumen de escorrentía incrementa en áreas impermeabilizadas (Rey Valencia y Zambrano Nájera, 2018). Por lo tanto, un mal manejo del suelo es un elemento condicionante de la susceptibilidad a la erosión (Alvarado García, 2020).

La degradación de los suelos a nivel mundial evidencia la necesidad de adoptar técnicas de manejo conservacionista, con el fin de mantener la cubierta vegetal para protegerlos del impacto de las gotas de lluvia y reducir su pérdida, de manera que las condiciones favorables de humedad y temperatura favorezcan la actividad biológica y la sucesión ecológica. Un suelo protegido es menos vulnerable a la erosión. Las plantas proveen un sostén mecánico a nivel subterráneo y una protección biofísica a nivel aéreo (Alvarado-García y Zúñiga-Amador, 2020).

Por ello, en suelos con riesgo bajo, la implementación de coberturas vivas y muertas, siembra con altas densidades, y cultivos asociados o de rotación son fundamentales para mejorar la infiltración del agua en el suelo. En suelos con pendientes fuertes y largas, las barreras vivas son la mejor garantía para reducir la acción erosiva de la escorrentía (Hincapié Gómez y Ramírez Ortíz, 2010), recuperar la fertilidad natural de los suelos e incrementar la diversidad macro y microbiológica (San Román, Cárdenas y CATIE, 2016).

\section{Prácticas de conservación del suelo}

La degradación de los suelos evidencia la necesidad de adoptar técnicas de manejo conservacionista que promuevan el uso de cubiertas vegetales (sobre todo, nativas) y un óptimo rendimiento biológico, químico y físico. Un suelo protegido es menos vulnerable a la erosión; por ello, a continuación, se describen algunas tecnologías de conservación de suelos:

\section{Abonos orgánicos}

Consisten en materiales de origen natural, producto de la descomposición de los desechos y que mejoran las condiciones físicas, químicas y microbiológicas del suelo. Pueden ser desechos de origen animal, vegetal o mixto; residuos de cultivos después de la cosecha; cultivos verdes (principalmente leguminosas); restos 
orgánicos agropecuarios (estiércol, purín); restos orgánicos agrícolas; compost, entre otros (Cajamarca, 2012).

La aplicación de materia orgánica humificada aporta nutrientes y mantiene la actividad microbiana; al incorporarla da como resultado una cantidad de beneficios importantes (Félix-Herrán, Sañudo-Torres, Rojo-Martínez, Martínez-Ruiz y OlaldePortugal,2008), por lo que, dentro de las ventajas que se podrían obtener con esta práctica está:, mejorar la estructura del suelo (agregados más estables y permeables); aumentar la retención de humedad del suelo, favorecer la disponibilidad de micronutrientes como el hierro, el cobre y el zinc; elevar la capacidad de intercambio catiónico a través de los ácidos húmicos; y controlar patógenos.

Como parte de los abonos orgánicos secos, se encuentran los abonos verdes. Un ejemplo de ello son los cultivos de cobertura de plantas leguminosas, que fijan el nitrógeno y lo incorporan al suelo para mejorar su fertilidad y calidad (Félix-Herrán et al., 2008; Herrera Izurieta, 2008).

Uno de los beneficios que obtiene el suelo con estos abonos es la cobertura permanente con material orgánico verde o seco, ya que un suelo desnudo es más sensible a la influencia directa del sol y la lluvia, por ende, a la erosión. Además, mejoran el microclima, reducen la incidencia de plagas con respecto a las plantaciones sin cobertura, aumentan la actividad biológica del suelo, mejoran las condiciones para el desarrollo radicular, e incrementan el rendimiento de los cultivos (FAO, 2018).

\section{Cultivos de cobertura}

Los cultivos de cobertura están caracterizados por presentar diversas funciones como la supresión de malezas, conservación de suelo y agua, control de plagas y enfermedades, alimentación humana y forraje. Es una técnica de bajo costo (reduce la necesidad de insumos externos), genera ingresos, incrementa la productividad y reduce la degradación de los recursos naturales (Pound, 1999).

En regiones tropicales lluviosas, los cultivos son de mayor importancia, ya que al ser establecidos con un cultivo de interés económico, lo protege contra la erosión o daños ocasionados por las lluvias. Además, aumentan la diversidad de insectos benéficos y reducen malezas. En términos generales, estos cultivos reducen la contaminación, la erosión, conservan la humedad y protegen la calidad de agua (Ligña-Sangucho, 2014).

\section{Barreras vivas}

Se siembran en hileras y en gran densidad, perpendicular a la pendiente (en contorno) o como complemento a una obra física. Son importantes en zonas de ladera, donde la erosión hídrica es evidente. Se combinan con cultivos de cobertura 
y otras prácticas de conservación de suelo y agua como las terrazas y barreras muertas. Se utilizan especies de rápido crecimiento como pastos, arbustos, árboles para uso forestal, árboles frutales, plantas leguminosas y otras (San Román, Cárdenas, \& CATIE, 2016).

Dentro de sus ventajas están: reducir la velocidad del agua de escorrentía y favorecer la infiltración; disminuir la degradación del suelo, prolongar el uso de la tierra, y propiciar la regeneración natural de vegetación secundaria (CruzFernández, 2005).

\section{Cortinas rompevientos}

Consisten en hileras de árboles que delimitan una propiedad o un potrero, con el fin de brindar protección contra el viento y producción de forraje, leña, madera, frutos, y postes. Además, estos fragmentos son significativos para la flora y fauna nativa, proporcionando hábitat y sirviendo como corredores biológicos. Una cerca compuesta por diferentes especies proporciona diversidad estructural, de modo que, proveen una mayor variedad de nichos para la fauna, y una mayor posibilidad de proveer alimento (como flores, néctar y frutas) para los animales a lo largo de todo el año (Alas-Martínez, 2007).

Bioingeniería

La bioingeniería se basa en la utilización de plantas vivas como elemento constructivo, con o sin material inerte (piedras, geotextiles o productos sintéticos). Esta técnica actúa como refuerzo, drenaje o barrera de sedimentos, a la vez que favorece el control de la erosión y la estabilización de taludes (Suárez, 2001; Mataix y López, 2007; García-Chevesich, 2015).

Algunas técnicas de la bioingeniería son:

- Hidrosiembra: consiste en la colocación de una mezcla acuosa y semillas sobre el suelo, utilizando equipos de bombeo a presión. Es un método sencillo, favorece la revegetación y es recomendable para inclinaciones de hasta $35^{\circ}$ (ca. $70 \%$ de pendiente).

- Estacas vivas: son cortes leñosos vivos (de herbáceas o arbustos) en forma de bisel, capaces de enraizar fácilmente y establecerse en pendientes moderadas $(<50 \%$ de inclinación).

- Fajinas vivas: son ramas vivas de plantas leñosas, atadas e instaladas sobre zanjas. Se recomiendan para pendientes moderadas ( $<50 \%$ de inclinación).

Revegetación

La vegetación representa la mejor protección contra la erosión; es multifuncional, económica y visualmente atractiva. El uso de especies nativas es la mejor garantía de un funcionamiento saludable del ecosistema y son una alternativa ideal desde el punto de vista ecológico, ético, estético y práctico. La cubierta vegetal actúa como 
un "colchón" protector entre el suelo y la atmósfera (Morgan, 2005) y su efectividad depende de la densidad de la cobertura superficial. Cuanto más cubierto de vegetación esté un terreno, menos propenso será a la erosión.

Dentro de las principales ventajas de la cubierta vegetal están: mejorar el aspecto del sitio, la calidad del suelo y del ecosistema en general; aportar servicios ecosistémicos; reducir la erosión; disminuir la velocidad de la escorrentía superficial; aumentar la infiltración del agua en el suelo; mejorar la calidad, estructura y resistencia mecánica del suelo; y proporcionar estabilidad, rugosidad y porosidad al suelo por medio de la materia orgánica (Alvarado-García, 2016; Alvarado García y Zúñiga Amador, 2020).

Existen especies pioneras con alta capacidad de tolerancia y resistencia que crecen y se establecen en terrenos intervenidos, degradados y pobres de nutrientes. Esto mejora sustancialmente las propiedades del suelo y contribuye al crecimiento de otras plantas; las raíces penetran el suelo compactado e incrementan la porosidad y la infiltración. Así, el proceso de rehabilitación es evidente y exitoso gracias sucesión ecológica (Alvarado García y Zúñiga Amador, 2020).

Un caso de estudio permitió evidenciar lo anterior durante el 2015 en el río Torres, ubicado en San José, Costa Rica. Para ello se pusieron a prueba tres herbáceas nativas: platanilla (Heliconia tortuosa Griggs), catalina (Dahlia imperialis Roezl ex Ortgies) y canutillo (Tradescantia zanonia (L.) Sw.), y tres arbustos nativos: zorrillo (Cestrum nocturnum L.), coralillo (Hamelia patens Jacq.) y quitirrisí (Lasianthaea fruticosa (L.) K.M. Becker). Esto permitió evaluar la producción de sedimentos en parcelas de $8 \mathrm{~m} 2$ y se observó que el grado de efectividad depende de la supervivencia de las plantas (ya sea por condiciones climáticas o del terreno) y de su capacidad para establecerse y retener sedimentos. Todas las especies fueron exitosas para el control de la erosión y eficaces para la rehabilitación ecológica de esos sitios (Alvarado y Zúñiga, 2018).

\section{CONCLUSIONES}

La erosión es el proceso predominante responsable de la pérdida y degradación de los suelos a nivel mundial, y su estudio es esencial para poder mitigar y prevenir la desertificación de las tierras. Por ello, el abordaje de este tema debe ser integral, de forma que se visualicen diversos escenarios, con diferente magnitud de impacto. Esta información es el insumo necesario para diseñar, definir e implementar acciones compatibles con la conservación del suelo, como las planteadas en este documento; lo cual dependerá del contexto, el tiempo y los recursos con los que se cuentan.

Ética, conflicto de intereses y declaración de financiamiento: la autora declara haber cumplido con todos los requisitos éticos y legales pertinentes, tanto durante el estudio como en el manuscrito; que no hay conflictos de interés de ningún tipo. Asimismo, la autora está de acuerdo con la versión editada final del documento. 


\section{REFERENCIAS}

Aiello, A., Adamo, M., \& Canora, F. (2015). Remote sensing and GIS to assess soil erosion with RUSLE3D and USPED at river basin scale in southern Italy. Catena, 131, 174-185. http://dx.doi.org/10.1016/j.catena.2015.04.003

Alas-Martínez, J. M. (2007). Barreras para la implementación de sistemas silvopastoriles y usos de suelo amigables con la biodiversidad en Matiguás, Nicaragua. (Tesis de Maestría). Centro Agronómico Tropical de Investigación y Enseñanza (CATIE), Turrialba, Costa Rica.

Almoza, Y., Medina, H., Schiettecatte, W., Ruiz, M. E., \& Leal, Z. (2007). Análisis de la relación temporal entre los valores de precipitación mensual y gastos en la subcuenca La Güira del río Cuyaguateje. Revista Ciencias Técnicas Agropecuarias, 16(4), 88-94.

Alvarado-García, V. (2016). La vegetación como factor de control de la erosión. Repertorio Científico, 19(1), 13-17.

Alvarado V., Bermúdez, T., Romero, M., \& Piedra, L. (2013). Plantas nativas para el control de la erosión en taludes de ríos urbanos. Spanish Journal of Soil Science, $4(1), 99-111$.

Alvarado-García, V. (2020). Modelación hidrológica del río Torres, San José, Costa Rica y su variación con respecto al cambio climático. (Tesis de Maestría). Centro Agronómico Tropical de Investigación y Enseñanza (CATIE), Turrialba, Costa Rica.

Alvarado, V., \& Zúñiga, M. A. (2018). Vegetación nativa como factor de control de erosión y restauración ecológica, San José, Costa Rica. Revista La Calera, 19(30), 39-47. https://doi.org/10.5377/calera.v18i30.7738

Alvarado García, V., \& Zúñiga Amador, M. A. (2020). Plantas nativas para el control de la erosión. Cartago, Costa Rica: Editorial Tecnológica de Costa Rica.

Álvarez Rojas, M., \& Peña, W. (2013). Estimación de niveles erosivos en suelos con cultivo de piña en Sarapiquí. Repertorio Científico, 16(1), 3-11.

Blanco Chávez, M. E. (2019). Erosión hídrica en la microcuenca del Cauce 31 de Diciembre, Nicaragua. Nexo Revista Científica, 32(2), 94-105. https://doi.org/10.5377/nexo.v32i02.9261

Cajamarca, D. (2012). Procedimientos para la elaboración de abonos orgánicos. (Tesis de Grado). Universidad de Cuenca, Cuenca, Ecuador.

Colotti Bizzarri, E. (1999). La erosividad: cualidad de la lluvia poco conocida. Terra Nueva Etapa, 15(24), 99-116.

Cruz-Fernández, M. (2005). Barreras vivas antierosivas para la agricultura de ladera de la Huasteca Potosina. San Luis Potosí, México: INIFAP-CIRNE.

Dlamini, P., Orchard, C., Jewitt, G., Lorentz, S., Titshall, L., \& Chaplot, V. (2011). Controlling factors of sheet erosion under degraded grasslands in the sloping lands of KwaZulu- Natal, South Africa. Agricultural Water Management, 98, 17111718.

Dumas Salazar, A. (2012). Riesgo de erosión hídrica en la cuenca hidrográfica del río Mundo (Tesis de Maestría). Universidad complutense de Madrid, Madrid, España. 
Durán V. H., Rodríguez, C. R., Martin, F. J., de Graaff, J., Francia, J. R., \& Flanagan, D. C. (2011). Environmental impact of introducing plant covers in the taluses of terraces: Implications for mitigating agricultural soil erosion and runoff. Catena, 84, 79-88.

Félix-Herrán, J., Sañudo-Torres, R., Rojo-Martínez, G., Martínez-Ruiz, R., \& OlaldePortugal, V. (2008). Importancia de los abonos orgánicos. Ra Ximhai, 4(1), 57-67. García, P. (2004). Interacciones entre la vegetación y la erosión hídrica. En: Valladares F. (Ed.). Ecología del bosque mediterráneo en un mundo cambiante (pp. 309334). EGRAF; Madrid, España.

García-Chevesich, P. (2015). Control de la erosión y recuperación de suelos degradados. Denver, USA: Outskirts Press

Gayoso J., \& Alarcón D. (1999). Guía de conservación de suelos forestales. Valdivia, Chile: Universidad Austral de Chile.

Henríquez, C. \& Cabalceta, G. (1999). Guía práctica para el estudio introductorio de los suelos con un enfoque Agrícola. San José, Costa Rica: Asociación Costarricense de la Ciencia del Suelo.

Henríquez, C., Ortiz, O., Largaespada, K., Portuguéz, P., Vargas, M., Villalobos, P., \& Gómez, D. (2011). Determinación de la resistencia a la penetración, al corte tangencial, densidad aparente y temperatura en un suelo cafetalero, Juan Viñas, Costa Rica. Agronomía Costarricense, 35(1), 175-184.

Hernández, D. (2011). Influencia de la pendiente y la precipitación en la erosión de taludes desprotegidos. (Tesis de Licenciatura). Universidad del Bio - Bio, Concepción, Chile.

Herrera Izurieta, F. (2008). Verdes gotas de vida. Manual de agricultura orgánica. Una alternativa sostenible para Galápagos. Islas Galápagos, Ecuador: FUNDAR Galápagos.

Hincapié Gómez, E., \& Ramírez Ortíz, F. A. (2010). Riesgo a la erosión en suelos de ladera de la zona cafetera. Cenicafé, Colombia: Fondo Nacional del Café.

León, J. (2001). Estudio y control de la erosión hídrica. Medellín, Colombia:

Universidad Nacional de Colombia.

Ligña-Sangucho, M. A. (2014). Efecto de cultivos de cobertura en el control de malezas y aporte de materia seca y nutrientes al suelo. (Tesis de Licenciatura). Escuela Agrícola Panamericana, Francisco Morazán, Honduras.

Mataix, C., \& López, J. (2007). Factores ambientales: funciones y uso de la vegetación en la estabilización de laderas. En: Confederación Hidrográfica del Ebro (Ed.). Zaragoza, España: Jornadas Técnicas sobre Estabilidad de Laderas en Embalse.

Mehuys, G. R., Tiessen, K. H. D., Villatoro, M., Sancho, F., \& Lobb, D. A. (2009). Erosión por labranza con arado de disco en suelos volcánicos de ladera en Costa Rica. Agronomía Costarricense, 33(2), 205-215.

Morgan, R. (2005). Soil erosion and conservation. Oxford, USA: National Soil Resources Institute - Cranfield University.

Muñoz Iniesta, D. J., Ferreira Ramírez, M. F., Escalante Arriaga, I. B., \& López García, J. (2013). Relación entre la cobertura del terreno y la degradación física y biológica de un suelo aluvial en una región semiárida. Terra Latinoamericana, 31 (3), 201-210. 
Núñez J. (2010). Manejo y conservación de suelos. San José, Costa Rica: EUNED. Organización de las Naciones Unidas para la Alimentación y la Agricultura, FAO.

(2018). Guía de buenas prácticas para la gestión y uso sostenible de los suelos en áreas rurales. Bogotá, Colombia: Organización de las Naciones Unidas para la Alimentación y la Agricultura y el Ministerio de Ambiente y Desarrollo Sostenible.

Pérez-Salinas, J. E., Rodríguez, F. R., López-Santos, A., Torres-González, J. A., MerazJiménez, A. de J., Díaz-Romo, A., \& Valdivia-Martínez, O. (2019). Erodabilidad y riesgo de erosión de suelos negros del centro de México: estudio de un Phaozem. Terra Latinoamericana, 37, 391-400. https://doi.org/10.28940/terra.v37i4.531

Pound, B. (1999). Cultivos de cobertura para la agricultura sostenible en América Latina. En: Sánchez, M. D., \& Rosales, M. (Eds.). Roma, Italia: Agroforestería para la producción animal en Latinoamérica.

Ramírez-Ortiz, F. A., Hincapié-Gómez, E., \& Sadeghian-Khalajabadi, S. (2009). Erodabilidad de los suelos de la zona central cafetera del departamento de Caldas. Cenicafé, 60(1), 58-71.

Rey Valencia, D. M., \& Zambrano Nájera, J. 2018. Estudio de la respuesta hidrológica en la cuenca urbana de montaña San Luis-Palogrande. Revista UIS Ingenierías, 17(1), 115-126. https://doi.org/10.18273/revuin. v17n1-2018011

Robinson, D. A., \& Woodun, J. K. (2008). An experimental study of crust development on chalk downland soils and their impact on runoff and erosion. European Journal of Soil Science, 59, 784-798.

Robredo-Sánchez, J. C. (2014). Cálculo de caudales de avenida. Madrid, España: Universidad Politécnica de Madrid.

San Román, L., Cárdenas, J., \& Centro Agronómico Tropical de Investigación y Enseñanza (CATIE). (2016). Buenas prácticas para el desarrollo de agricultura sostenible y afrontar el cambio climático. Vásquez de Coronado, Costa Rica: Programa Regional de Investigación e Innovación por Cadenas de Valor Agrícola, PRIICA.

Sepúlveda-Lozada, A., Geissen, V., Ochoa-Gaona, S., Jarquín-Sánchez, A., Hernández de la Cruz, S., Capetillo, E., \& Zamora-Cornelio, L. F. (2009). Influencia de tres tipos de vegetación ribereña en el control de la erosión fluvial en Pantanos de Centla, México. Revista de Biología Tropical, 57(4), 11531163.

Suárez, F. (1980). Conservación de suelos. San José, Costa Rica: Editorial IICA.

Suárez, J. (2001). Control de erosión en zonas tropicales. Bucaramanga, Colombia: Universidad Industrial de Santander.

Viramontes, D., Descroix, L., \& Bollery, A. (2006). Variables de suelos determinantes del escurrimiento y la erosión en un sector de la Sierra Madre Occidental. Ingeniería hidráulica en México, 21(1), 73-83. 